Pacific Journal of 


\title{
SOME TOPOLOGICAL PROPERTIES OF PIERCING POINTS
}

\author{
D. R. McMillan, JR.
}

Let $K$ be the closure of one of the complementary domains of a 2-sphere $S$ topologically embedded in the 3-sphere, $S^{3}$. We give first (Theorem 1) a characterization of those points $p \in S$ with the following property: there exists a homeomorphism $h: K \rightarrow S^{3}$ such that $h(S)$ can be pierced with a tame arc at $h(p)$. The topological property of $K$ which distinguishes such a "piercing point" $p$ is this: $K-p$ is 1-ULC. Using this result, we find (Theorems 2 and 3 ) that $p$ is a piercing point of $K$ if and only if $S$ is arcwise accessible at $p$ by a tame arc from $S^{3}-K$ (note: perhaps $S$ cannot be pierced with a tame arc at $p$, even if $p$ is a piercing point of $K$ ). Thus, the "tamely arcwise accessible" property is independent of the embedding of $K$ in $S^{3}$. The corollary to Theorem 2 gives an alternate proof of an as yet unpublished fact, first proven by R. H. Bing: a topological 2-sphere in $S^{3}$ is arcwise accessible at each point by a tame arc from at least one of its complementary domains.

In the last section of the paper, we give two applications of the above theorems. First, we show in Theorem 4 that $S$ can be pierced with a tame arc at $p$ if and only if $p$ is a piercing point of both $K$ and the closure of $S^{3}-K$. Finally, we remark in Theorem 5 that $S$ can be pierced with a tame arc at each of its points if it is "free" in the sense that for each $\varepsilon>0, S$ can be mapped into each of its complementary domains by a mapping which moves each point less than $\varepsilon$. It is not known whether each 2 -sphere $S$ with this last property is tame.

A space homeomorphic to such a set $K$ in $S^{3}$ (as described at the beginning of the Introduction) is called a crumpled cube. We write Bd $K=S$ and Int $K=K-\mathrm{Bd} K$. An arc $A$ in $S^{3}$ is said to pierce a 2-sphere $S$ in $S^{3}$ if $A \cap S$ is an interior point $p$ of $A$ and the two components of $A-p$ lie in different components of $S^{3}-S$. The piercing points of a crumpled cube are defined as above and were first considered by Martin [10]. It follows from Lemmas 2 and 3 of [10] and [6; Th. 11] that the nonpiercing points of a crumpled cube $K$ form a O-dimensional $F_{\sigma}$ subset of $\mathrm{Bd} K$.

If $C$ and $D$ are subsets of a space $Y$ with metric $d$, and $\varepsilon>0$, we use $B(C, D ; \varepsilon)$ to denote the set of all points $x \in D$ such that for some $y \in C, d(x, y)<\varepsilon$. The metric on $E^{3}$ and $S^{3}$ is always assumed to be the ordinary Euclidean one. Let $\Delta^{n}(n \geqq 1)$ denote a closed $n$ - 
simplex. If $Y$ is a metric space and $A \subset Y$, we say that $A$ is $n$-LC $(n \geqq 0)$ at $p \in \mathrm{Cl} A \subset Y(\mathrm{Cl} A=$ the closure of $A)$ if for each $\varepsilon>0$ there is a $\delta>0$ such that each mapping of $\mathrm{Bd} \Delta^{n+1}$ into $B(p, A ; \delta)$ extends to a mapping of $\Delta^{n+1}$ into $B(p, A ; \varepsilon)$. We say that $A$ is $n$ $\mathrm{ULC}(n \geqq 0)$ if for each $\varepsilon>0$ there is a $\delta>0$ such that each mapping of $\mathrm{Bd} \Delta^{n+1}$ into a subset of $A$ of diameter less than $\delta$ extends to a mapping of $\Delta^{n+1}$ into a subset of $A$ of diameter less than $\varepsilon$. We refer to a mapping $f: \mathrm{Bd} \Delta^{2} \rightarrow Y$ as a loop.

By a null sequence of subsets of a metric space, we mean one such that the diameters of its elements converge to zero. A Sierpinski curve $X$ is (uniquely) defined as any space homeomorphic to $\left[\mathrm{Bd} \Delta^{3}\right]-\mathrm{U}$ Int $D_{i}$, where $D_{1}, D_{2}, \cdots$, is a null sequence of disjoint 2-cells whose union is a dense subset of $\mathrm{Bd} \Delta^{3}$. The inaccessible part of $X$ corresponds to $\left[\mathrm{Bd} \Delta^{3}\right]-\mathrm{UD}_{i}$. For a more detailed discussion of Sierpinski curves, see [3].

2. Preliminary lemmas. The following is Theorem 1 of [12], stated here for the reader's convenience.

Lemma 1. Let $C$ be a q-cell $(q=1,2$, or 3) topologically embedded in $E^{3}$, and let $D \subset \mathrm{Bd} C$ be a $(q-1)$-cell. Let $A_{1}, A_{2}, \cdots, A_{k}$ be a finite disjoint collection of tame arcs in $E^{3}-D$ with each $\mathrm{Bd}$ $A_{i} \subset E^{3}-C$. Then, there exists a compact set $E \subset C-D$ such that, for each $\varepsilon>0$, there is a homeomorphism $h: E^{3} \rightarrow E^{3}$ with each $h\left(A_{i}\right) \subset$ $E^{3}-C$ and $h$ is the identity outside the $\varepsilon$-neighborhood of $E$.

We shall also need the following [5; Th. 2].

Lemma 2. Let $B$ be a closed subset of $\Delta^{2}$; let $A$ be a subset of the separable metric space $Y$ and suppose that $A$ is O-LC and 1-LC at each point of $Y$. Let $\varepsilon>0$ and a mapping $f: \Delta^{2} \rightarrow \mathrm{Cl} A$ be given. Then, There is a mapping $f^{*}: \Delta^{2} \rightarrow \mathrm{Cl} A$ such that

$$
f^{*}\left(\Delta^{2}-B\right) \subset A, f^{*}|B=f| B, \text { and } d\left(f^{*}(x), f(x)\right)<\varepsilon
$$

for each $x \in \Delta^{2}$, where $d$ is the metric for $Y$.

Let $X$ be a topological space, and $Y$ a closed subset of $X$. A loop $f: \mathrm{Bd} \Delta^{2} \rightarrow X$ will be said to be contractible in $X(\bmod Y)$ if there exists a connected open set $N$ in $\Delta^{2}$ such that $\mathrm{Bd} \Delta^{2} \subset N$, and a mapping $F: \mathrm{Cl} N \rightarrow X$ such that $F \mid \mathrm{Bd} \Delta^{2}=f$, and $F$ maps the (point-set) boundary of $N$ (in $\Delta^{2}$ ) into $Y$.

Lemma 3. Let $K$ be a crumpled cube in $S^{3}$, and let $U$ be an 
open subset of $K$ such that $U \cap \mathrm{Bd} K$ is an open 2-cell $T$. Let $A$ be a compact subset of $K$ such that $A \cap \mathrm{Bd} K$ consists of a single point $p$ in $T$, where $K^{*}-p$ is 1-LC at $p$ and $K^{*}$ is the crumpled cube $S^{3}$ - Int $K$. Then, if a loop in $U-A$ is contractible in $U-A$ $(\bmod T-p)$, it is contractible to a point in $(U-A) \cup(W-A)$, where $W$ is any open set in $S^{3}$ containing $p$.

Proof. Let $N$ be a connected open set in $\Delta^{2}$ containing $\mathrm{Bd} \Delta^{2}$, let $W$ be an open set in $S^{3}$ containing $p$, and let

$$
F: \mathrm{Cl} N \rightarrow U-A
$$

be a mapping which takes the boundary $B$ of $N$ in $\Delta^{2}$ into $T-p$. By the homotopy extension theorem, $F \mid B: B \rightarrow T$ extends to a mapping $G: \Delta^{2} \rightarrow T$. Hence, by Lemma 2 , and the fact that $K^{*}-p$ is 1-LC at $p, F \mid B$ extends to

$$
G^{*}: \Delta^{2} \rightarrow[T-p] \cup\left[\left(W \cap K^{*}\right)-p\right] .
$$

Finally, define $H: \Delta^{2} \rightarrow(U-A) \cup(W-A)$ by $H|\mathrm{Cl} N=F| \mathrm{Cl} N$ and $H\left|\Delta^{2}-N=G^{*}\right| \Delta^{2}-N$. Then $H$ is the required contraction of $F \mid \operatorname{Bd} \Delta^{2}$.

REMARK. Given the notation of the lemma, and a loop $f: \mathrm{Bd} \Delta^{2}$ $\rightarrow U-A$, a necessary condition for $f$ to be contractible to a point in $(U-A) \cup(\mathrm{W}-A)$, where $W$ is a small neighborhood of $p$ in $S^{3}$, is that $f$ be contractible in $U-A(\bmod T-p)$.

\section{Characterizations of piercing points.}

THEOREM 1. Let $K$ be a crumpled cube and $p$ a point of $\mathrm{Bd} K$. Then $p$ is a piercing point of $K$ if and only if $K-p$ is 1-LC at $p$.

Proof. We may assume, by [8] and [9], that $K$ is embedded in $S^{3}$ in such a manner that there exists a homeomorphism $h$ of $C$, the closure of $S^{3}-K$, onto the closed unit ball in $E^{3}$. Let $A$ be the inverse image under $h$ of the straight line segment in $E^{3}$ from the origin to $h(p)$. Then $A$ is an arc which is locally tame in $S^{3}$ except possibly at $p$, and according to Martin [10], $p$ is a piercing point of $K$ if and only if $A$ is tame. By [11, Lemma 5], $A$ is tame if and only if $S^{3}-A$ is 1-LC at $p$. Hence the problem is reduced to showing that $S^{3}-A$ is 1-LC at $p$ if and only if $K-p$ is 1-LC at $p$.

We shall give the details of the "if" part of the above assertion. The converse is merely a rearrangement of the same ideas. Suppose 
$K-p$ is 1-LC at $p$, and let $\varepsilon$ be a positive number. We must find a $\delta>0$ such that each loop in $\mathrm{B}\left(p, S^{3}-A ; \delta\right)$ is contractible in $B\left(p, S^{3}-A ; \varepsilon\right)$. We assume that $\varepsilon$ is less than the distance from $p$ to $h^{-1}((0,0,0))$. Since $K-p$ is $1-\mathrm{LC}$ at $p$, there exists $\rho>0$ such that each loop in $B(p, K-p ; \rho)$ is contractible in $B(p, K-p ; \varepsilon)$. Let $U$ be an open subset of $S^{3}$ such that $p \in U \subset B\left(p, S^{3} ; \rho\right)$ and such that there is a homeomorphism of $U \cap C$ onto the set of points in $E^{3}$ having nonnegative $z$-coordinates which takes $U \cap A$ into the $z$-axis. Finally, choose $\delta>0$ so that $B\left(p, S^{3} ; \delta\right) \subset U$.

Now, a given loop in $B\left(p, S^{3}-A ; \delta\right)$ is homotopic in $U-A$ to a loop in

$$
(U \cap K)-p \subset B(p, K-p ; \rho),
$$

and this loop in turn is contractible to a point in $B(p, K-p ; \varepsilon)$, as required.

REMARK. Since $K$ is compact and locally contractible, the condition " $K-p$ is $1-\mathrm{LC}$ at $p$ " is equivalent to " $K-p$ is 1 -ULC".

CoROLlaRY. Let $K$ be a crumpled cube, and $p$ a point of $S=$ $\mathrm{Bd} K$. Then $p$ is a piercing point of $K$ if and only if the following condition holds: For each $\varepsilon>0$, there is a $\delta>0$ such that each simple closed curve in $B(p, S-p ; \delta)$ is contractible in $B(p, K-p ; \varepsilon)$.

Proof. The condition is necessary by the preceding theorem. To show sufficiency, assume the notation of the preceding proof and let $\varepsilon>0$ be given as before. Let $\delta>0$ be chosen to satisfy the above condition and so that only the component of $A-B\left(p, S^{3} ; \delta\right)$ which contains $h^{-1}((0,0,0))$ fails to lie in $B\left(p, S^{3} ; \varepsilon\right)$. We also assume that $A$ is locally polyhedral at each point of $A-p$. Then, each piecewiselinear homeomorphism

$$
f: \mathrm{Bd} \Delta^{2} \rightarrow B\left(p, S^{3}-A ; \delta\right)
$$

extends to a piecewise-linear mapping $F$ of $\Delta^{2}$ into $B\left(p, S^{3}-p ; \delta\right)$ such that $F$ is in general position relative to $A$. Hence $F^{-1}(A)$ is finite. If $x \in F^{-1}(A)$, then $F$ restricted to a sufficiently small curve enclosing $x$ represents a loop in $B\left(p, S^{3}-A ; \delta\right)$ which is homotopic in $B\left(p, S^{3}-\right.$ $A ; \varepsilon)$ to a loop in $B(p, S-p ; \delta)$, and hence is contractible in $B(p, K-$ $p ; \varepsilon)$. This permits us to redefine $F$ in a small neighborhood of each $x \in F^{-1}(A)$, and thus obtain an extension of $f$ mapping $\Delta^{2}$ into $B\left(p, S^{3}-\right.$ $A ; \varepsilon)$. Hence $S^{3}-A$ is 1 -LC at $p$ and the result follows. 
point of the crumpled cube $K^{*}=S^{3}-\operatorname{Int} K$. Suppose $A$ is an arc in $K$ having $p$ as an end-point, such that $A \cap S=p$, where $S=\mathrm{Bd}$ $K$. If there exists a homeomorphism $h: K \rightarrow S^{3}$ such that $h(A)$ is tame, then $A$ is tame.

Proof. Since $h(A)$ is tame, $A$ is locally tame in $S^{3}$ except possibly at $p$. Hence, by [11; Lemma 5], it suffices to show that $S^{3}-A$ is 1-LC at $p$. Suppose $\varepsilon>0$. Let $U$ be an open set in $S^{3}$ such that $p \in U \subset B\left(p, S^{3} ; \varepsilon\right)$ and $U \cap S$ is an open 2-cell $T$. Since $h$ is a homeomorphism, and since $S^{3}-h(A)$ is 1-LC at $h(p)$, there exists $\rho>0$ such that each loop in $B(p, K-A ; \rho)$ is contractible in $(U \cap K)-A$ $(\bmod T-p)$. Choose $\mu>0$ so that each loop in $B\left(p, K^{*} ; \mu\right)$ is contractible in $B\left(p, K^{*} ; \rho\right)$. Finally, let $\delta>0$ be such that each pair of points in $B(p, S ; \delta)$ can be joined by an arc in $B(p, S ; \mu)$.

Now let a loop in $B\left(p, S^{3}-A ; \delta\right)$ be given. We give here an outline of the proof that this loop is contractible in $B\left(p, S^{3}-A ; \varepsilon\right)$. The details are left to the reader. There are three steps:

1. After performing a small homotopy in $B\left(p, S^{3}-A ; \delta\right)$, we assume that this loop is a simple closed curve $J$ such that $J \cap K^{*}$ consists of a finite number of disjoint $\operatorname{arcs} L_{1}, L_{2}, \cdots, L_{k}$, with $L_{i} \cap$ $S=\operatorname{Bd} L_{i}$, for each $i$.

2. For each $i$, let $Z_{i}$ be an arc in $B(p, S ; \mu)-p$ joining the endpoints of $L_{i}$. Then $L_{i}$ is homotopic in $B\left(p, K^{*} ; \rho\right)$, with end-points fixed, to $Z_{i}$. Since $K^{*}-p$ is 1-LC at $p$, Lemma 2 allows us to adjust this homotopy to give one in $B\left(p, K^{*} ; \rho\right)-p$ between $L_{i}$ and $Z_{i}$. Hence, by piecing together these homotopies, we see that the given loop is homotopic in $B\left(p, S^{3}-A ; \rho\right)$ to the loop

$$
\left[J-\cup \operatorname{Int} L_{i}\right] \cup \cup Z_{i}
$$

in $B(p, K-A ; \rho)$.

3. This last loop is contractible in $(U \cap K)-A(\bmod T-p)$. Hence, by Lemma 3 , it is contractible to a point in $B\left(p, S^{3}-A ; \varepsilon\right)$. This completes the proof.

REMARK. Using the same techniques, and Lemma 3, we could prove this lemma with "tame" replaced consistently by "cellular" or "has a simply-connected complement in $S^{3}$ " everywhere in its statement. In these two alternate formulations, we could permit $A$ to be any compact absolute retract, and $p$ any point of $A$.

THEOREM 2. Let $K$ be a crumpled cube in $S^{3}$, and $p$ a point of $S=\operatorname{Bd} K$. If $p$ is a piercing point of $K$, then there is a tame arc $A$ in $K^{*}=S^{3}-$ Int $K$ having $p$ as an end-point such that $A \cap S=p$. 
Proof. By Lemma 4, it suffices to show that there is an arc $A$ in $K^{*}$ having $p$ as an end-point such that $A \cap S=p$, and such that for some embedding $h: K^{*} \rightarrow S^{3}, h(A)$ is tame. We choose $h$ so that the closure of $S^{3}-h\left(K^{*}\right)$ is a 3-cell ([8] and [9]). Hence, the theorem will follow as stated above if we can prove it in the special case when $K$ is a closed 3-cell. We make this assumption to simplify the notation.

Let $f$ be a homeomorphism of the closed unit ball $B$ in $E^{3}$ onto $K$, with $f((0,0,1))=p$. Let $T_{i}(i=1,2, \cdots)$ be the 2-cell which is the $f$-image of the intersection of $B$ with the plane $z=1-1 / i$. Let the 3-cell $C_{i}(i=1,2, \cdots)$ be defined inductively as follows: $C_{1}$ is the closure of the component of $K-T_{1}$ not containing $p ; C_{i}(i \geqq 2)$ is the closure of the component of

$$
K-T_{i}-\bigcup_{j<i} C_{j}
$$

not containing $p$. Finally, let $A^{*}$ be a tame arc in $S^{3}$ having $p$ as one end-point and the other end-point not in $K$. We assume that $A^{*} \cap C_{1}=\phi$.

According to Lemma 1 , there is for each $i>1$, a homeomorphism $g_{i}: S^{3} \rightarrow S^{3}$ which is the identity outside a small neighborhood $U_{i}$ of $T_{i}$ and which is such that $g_{i}\left(A^{*}\right) \cap T_{i}=\phi$. In particular, the $U_{i}^{\prime} \mathrm{s}$ may be chosen to form a null sequence of disjoint sets. Let $g$ be the homeomorphism of $S^{3}$ onto itself which agrees with $g_{i}$ on $U_{i}$, for each $i$, and otherwise is the identity. Then $g\left(A^{*}\right) \cap T_{i}=\phi$, for each $i$, and $g(p)=p$.

Again using Lemma 1, there is, for each $i>1$, a compact set $E_{i} \subset C_{i}-\left(T_{i} \cup T_{i-1}\right)$ (by the previous paragraph, there is a 2-cell in Bd $C_{i}$ containing $T_{i} \cup T_{i-1}$ and missing $g\left(A^{*}\right)$ ) and a homeomorphism $k_{i}: S^{3} \rightarrow S^{3}$ which is the identity outside an arbitrarily small neighborhood $V_{i}$ of $E_{i}$ and which is such that $k_{i} g\left(A^{*}\right) \cap C_{i}=\phi$, for each $i$. We choose $V_{i}$ so close to $E_{i}$ that the $V_{i}$ 's form a null sequence of disjoint sets, and so that $V_{i}$ misses the closure of $K-C_{i}$. Let $k$ be the homeomorphism of $S^{3}$ onto itself which agrees with $k_{i}$ on $V_{i}$, for each $i$, and reduces to the identity otherwise. Then $A=k g\left(A^{*}\right)$ is the required arc.

Corollary (Bing). A topological 2-sphere in $S^{3}$ is arcwise accessible at each point by a tame arc from at least one of its complementary domains.

Proof. Let $K$ and $K^{*}$ be the two crumpled cubes into which the 2-sphere $S$ decomposes $S^{3}$. If $p \in S$, then either $p$ is a piercing point of $K$, or $p$ is a piercing point of $K^{*}([10$; Theorem $])$. The result 
then follows from the preceding theorem.

THEOREM 3. Let $K$ be a crumpled cube in $S^{3}$, and $p$ a point of $S=\operatorname{Bd} K$. If there is a tame arc $A$ in $K^{*}=S^{3}-$ Int $K$ having $p$ as an end-point and such that $A \cap S=p$, then $p$ is a piercing point of $K$.

Proof. It suffices to establish the condition given in the corollary to Theorem 1. Thus, take $\varepsilon>0$. We assume that $\varepsilon$ is less than the distance between $p$ and $q$, where $q$ is the other end-point of $A$. Choose $\delta>0$ so that $B(p, S ; \delta)$ lies interior to a closed 2-cell $D \subset$ $B(p, S ; \varepsilon)$.

Since $A$ is locally tame at $p$, there is a tame 2 -sphere

$$
Z^{*} \subset B\left(p, S^{3} ; \delta\right)
$$

which separates $p$ from $q$ in $S^{3}$ and which meets $A$ at precisely one point $r \in \operatorname{Int} A$, at which $A$ pierces $Z^{*}$. Let $T$ be a small closed 2cell in $Z^{*}$ missing $K$ and such that $r \in \operatorname{Int} T$. Note that, by linking considerations, $\mathrm{Bd} T$ is not contractible in $B\left(p, K^{*} ; \varepsilon\right)-A$.

Appealing to [2; Th. 1] and [4; Th. 1], we obtain, for each $\rho>0$, a tame Sierpinski curve $X \subset S$ such that each component $U_{i}$ $(i=1,2, \cdots)$ of $S-X$ has diameter less than $\rho$, and a homeomorphism $h: S^{3} \rightarrow S^{3}$ which moves each point of $S^{3}$ less than $\rho$, which is the identity outside $B\left(Z^{*} \cap S, S^{3} ; \rho\right)$, and which is such that $h\left(Z^{*}\right) \cap X$ consists of a finite disjoint collection of simple closed curves each in the inaccessible part of $X$. Let $Z=h\left(Z^{*}\right)$. By choosing $\rho$ sufficiently small, we may ensure that $h$ is the identity on $T$ and that $Z$ retains all the properties originally required of $Z^{*}$. A final requirement on $\rho$ is that $\rho<\varepsilon-\delta$ and that the component of $S-X$ containing $p$ should not meet $Z$ (if $p \in X$, then $S$ can be pierced with a tame are at $p$, by [6; Th. 6]).

We assert that there is at least one component of $Z \cap S$ separating $p$ from $\mathrm{Bd} D$ in $D$ (this component is necessarily a simple closed curve). If not, then $Z \cap X$ consists of a finite number of simple closed curves each of which is contractible in $D-p$, and $Z \cap(S-X)$ can be covered by the null sequence of disjoint open 2-cells of diameter less than $\rho$ in $S: U_{1}, U_{2}, \cdots$. Note that $U_{i} \cap Z$ is compact. It is now easy, using the homotopy extension theorem on each of the inclusions $U_{i} \cap Z \rightarrow U_{i}$ as in the proof of Lemma 3 , to construct a mapping contracting $\mathrm{Bd} T$ in

$$
\left[K^{*} \cap(Z-\operatorname{Int} T)\right] \cup[B(p, S-p ; \varepsilon)] \subset B\left(p, K^{*} ; \varepsilon\right)-A,
$$

a contradiction. 
By the preceding paragraph, we may let $L$ be an innermost (in $Z-T$ ) one of the components of $S \cap Z$ which separates $p$ from $\mathrm{Bd}$ $D$ in $D$. Let $L$ bound the 2-cell $F \subset Z-T$. Note that $L$ is not contractible in $B\left(p, K^{*} ; \varepsilon\right)-A$ and that no component of $S \cap \operatorname{Int} F$ separates $p$ from $\mathrm{Bd} D$ in $D$. Hence, by the argument of the preceding paragraph, the "large" component of $F-S$ lies in Int $K$, and $L$ is contractible in

$$
[K \cap F] \cup[B(p, S-p ; \varepsilon)] \subset B(p, K-p ; \varepsilon) .
$$

Since each simple closed curve in $B(p, S-p ; \delta)$ is homotopic in $D-p$ to $L$, the proof is complete.

\section{Some applications,}

THEOREM 4. Let $S$ be a 2-sphere topologically embedded in $S^{3}$, and let $K$ and $K^{*}$ be the two crumpled cubes into which $S$ divides $S^{3}$. Then $S$ can be pierced with a tame arc at a point $p \in S$ if and only if $p$ is a piercing point of $K$ and a piercing point of $K^{*}$.

Proof. The "only if" part of the theorem follows from Theorem 3. For the converse, suppose that $p$ is a piercing point of each of $K$ and $K^{*}$, and let $A$ be an arc in $S$ such that $A$ is locally tame except possibly at the end-point $p$. By [6; Th. 6], $S$ can be pierced with a tame arc at $p$ if $A$ is tame.

To show that $A$ is tame, we proceed in essentially the same manner as in the proof of [6; Lemma 6.1]. That is, let $S^{\prime}$ be a 2-sphere in $S^{3}$ which contains $A$ and is locally tame at each point of $S^{\prime}-A$, and which is homeomorphically so close to $S$ that $p$ is a piercing point of each of the crumpled cubes $L$ and $L^{*}$ into which $S^{\prime}$ divides $S^{3}$ (use Theorems 2 and 3). It suffices to show that $S^{\prime}$ is tame.

Exactly as in [6], $S^{\prime}$ is locally tame at each point of $A-p$. Hence, $S^{\prime}$ is locally tame except possibly at $p$. It follows easily, since $L-p$ and $L^{*}-p$ are each $1-\mathrm{LC}$ at $p$, that $S^{3}-S^{\prime}$ is 1-LC at each point of $S^{\prime}$ and hence that $S^{\prime}$ is tame by [1; Th. 6]. This completes the proof.

In [7], Hempel studied the properties of a surface $S(=\mathrm{Bd} K)$ which is free relative to one of its complementary domains (Int $K$ ) in $S^{3}$ (i.e., $S$ satisfies the mapping condition stated in the following theorem). It is not known whether the crumpled cube of this theorem is necessarily a 3-cell.

Theorem 5. Let $K$ be a crumpled cube, and let $S=\mathrm{Bd} K$. Suppose that for each $\varepsilon>0$ there exists a mapping $f: S \rightarrow$ Int $K$ which 
moves each point of $S$ less than $\varepsilon$. Then each point of $S$ is a piercing point of $K$.

Proof. We shall verify the condition given in the corollary to Theorem 1. Suppose $p \in S$ and $\varepsilon>0$. Choose $\delta>0$ so that there is a closed 2-cell $D \subset S$ such that

$$
B(p, S ; \delta) \subset D \subset B(p, S ; \varepsilon) .
$$

Then, if $J$ is a simple closed curve in $B(p, S-p ; \delta)$ bounding a 2cell $D^{*} \subset D$, there is a $\rho>0$ such that $\rho$ is less than the distance from $D$ to the complement of $B(p, K ; \varepsilon)$ and such that each mapping of $J$ into $K$ which moves each point of $J$ less than $\rho$ is homotopic in $B(p, K-p ; \delta)$ to the inclusion of $J$ into $B(p, K-p ; \delta)$.

Suppose $f: S \rightarrow$ Int $K$ is a mapping which moves each point of $S$ less than $\rho$. Then $J$ is homotopic in $B(p, K-p ; \delta)$ to $f(J)$, and $f(J)$ bounds the singular 2-cell

$$
f\left(D^{*}\right) \subset B(p, K ; \varepsilon)-S .
$$

This completes the proof.

REMARK. If $S \subset S^{3}$ is a topological 2-sphere which is free relative to each of its complementary domains, then it follows from the foregoing theorems that $S$ can be pierced with a tame arc at each of its points.

\section{REFERENCES}

1. R. H. Bing, A surface is tame if its complement is 1-ULC, Trans. Amer. Math. Soc. 101 (1961), 294-305.

2. _ - Each disk in $E^{3}$ contains a tame arc, Amer. J. Math. 84 (1962), 583-590.

3. ___ Pushing a 2-sphere into its complement, Michigan Math. J. 11 (1964), $33-45$.

4. _ Improving the intersections of lines and surfaces, Michigan Math. J. 14 (1967), 155-159.

5. S. Eilenberg and R. L. Wilder, Uniform local connectedness and contractibility, Amer. J. Math. 64 (1942), 613-622.

6. D. S. Gillman, Side approximation, missing an arc, Amer. J. Math. 85 (1963), 459-476.

7. John Hempel, Free surfaces in $S^{3}$ (to appear).

8. N. Hosay, The sum of a real cube and a crumpled cube is $S^{3}$ (corrected title), Abstract 607-17, Notices Amer. Math. Soc. 10 (1963), 666.

9. L. L. Lininger, Some results on crumpled cubes, Trans. Amer. Math. Soc. 118 (1965), 534-549.

10. Joseph Martin, The sum of two crumpled cubes, Michigan Math. J. 13 (1966), $147-151$.

11. D. R. McMillan, Jr., Local properties of the embedding of a graph in a 3-manifold, Canad. J. Math. 18 (1966), 517-528.

12. - A criterion for cellularity in a manifold, II, Trans. Amer. Math. Soc. 126 (1967), 217-224. 
Received July 25, 1966. This research was supported in part by Grant NSF GP-4125. The author is an Alfred P. Sloan Fellow.

The UNIVERSITY OF Virginia

Charlottesville, Virginia 


\section{PACIFIC JOURNAL OF MATHEMATICS}

\section{EDITORS}

\section{H. SAMELSON}

Stanford University

Stanford, California

J. P. JANS

University of Washington

Seattle, Washington 98105

\section{J. DugundJI}

University of Southern California Los Angeles, California 90007

RICHARD ARENS

University of California

Los Angeles, California 90024

\section{ASSOCIATE EDITORS}

E. F. BECKENBACH
B. H. NeUmanN

\section{SUPPORTING INSTITUTIONS}

UNIVERSITY OF BRITISH COLUMBIA CALIFORNIA INSTITUTE OF TECHNOLOGY

UNIVERSITY OF CALIFORNIA

MONTANA STATE UNIVERSITY

UNIVERSITY OF NEVADA

NEW MEXICO STATE UNIVERSITY

OREGON STATE UNIVERSITY

UNIVERSITY OF OREGON

OSAKA UNIVERSITY

UNIVERSITY OF SOUTHERN CALIFORNIA

\author{
STANFORD UNIVERSITY \\ UNIVERSITY OF TOKYO \\ UNIVERSITY OF UTAH \\ WASHINGTON STATE UNIVERSITY \\ UNIVERSITY OF WASHINGTON \\ AMERICAN MATHEMATICAL SOCIETY \\ CHEVRON RESEARCH CORPORATION \\ TRW SYSTEMS \\ NAVAL ORDNANCE TEST STATION
}

Mathematical papers intended for publication in the Pacific Journal of Mathematics should be typewritten (double spaced). The first paragraph or two must be capable of being used separately as a synopsis of the entire paper. It should not contain references to the bibliography. Manuscripts may be sent to any one of the four editors. All other communications to the editors should be addressed to the managing editor, Richard Arens at the University of California, Los Angeles, California 90024.

50 reprints per author of each article are furnished free of charge; additional copies may be obtained at cost in multiples of 50 .

The Pacific Journal of Mathematics is published monthly. Effective with Volume 16 the price per volume ( 3 numbers) is $\$ 8.00$; single issues, $\$ 3.00$. Special price for current issues to individual faculty members of supporting institutions and to individual members of the American Mathematical Society: $\$ 4.00$ per volume; single issues $\$ 1.50$. Back numbers are available.

Subscriptions, orders for back numbers, and changes of address should be sent to Pacific Journal of Mathematics, 103 Highland Boulevard, Berkeley 8, California.

Printed at Kokusai Bunken Insatsusha (International Academic Printing Co., Ltd.), 7-17, Fujimi 2-chome, Chiyoda-ku, Tokyo, Japan.

\section{PUBLISHED BY PACIFIC JOURNAL OF MATHEMATICS, A NON-PROFIT CORPORATION}

The Supporting Institutions listed above contribute to the cost of publication of this Journal, but they are not owners or publishers and have no responsibility for its content or policies. 


\section{Pacific Journal of Mathematics}

\section{Vol. 22, No. $2 \quad$ February, 1967}

Paul Frank Baum, Local isomorphism of compact connected Lie groups ....

Lowell Wayne Beineke, Frank Harary and Michael David Plummer, On the

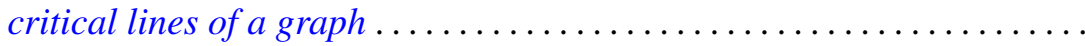

Larry Eugene Bobisud, On the behavior of the solution of the telegraphist's equation for large velocities .......................... 213

Richard Thomas Bumby, Irreducible integers in Galois extensions . . . . . . 221

Chong-Yun Chao, A nonimbedding theorem of nilpotent Lie algebras ..... 231

Peter Crawley, Abelian p-groups determined by their Ulm sequences ...... 235

Bernard Russel Gelbaum, Tensor products of group algebras ........... 241

Newton Seymour Hawley, Weierstrass points of plane domains .......... 251

Paul Daniel Hill, On quasi-isomorphic invariants of primary groups . . . . . 257

Melvyn Klein, Estimates for the transfinite diameter with applications to confomral mapping ................................ 267

Frederick M. Lister, Simplifying intersections of disks in Bing's side approximation theorem ............................. 281

Charles Wisson McArthur, On a theorem of Orlicz and Pettis ........... 297

Harry Wright McLaughlin and Frederic Thomas Metcalf, An inequality for

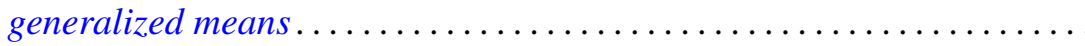

Daniel Russell McMillan, Jr., Some topological properties of piercing

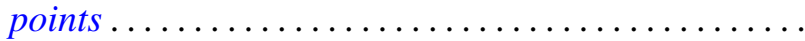

Peter Don Morris and Daniel Eliot Wulbert, Functional representation of topological algebras .

Roger Wolcott Richardson, Jr., On the rigidity of semi-direct products of Lie algebras..................................

Jack Segal and Edward Sandusky Thomas, Jr., Isomorphic

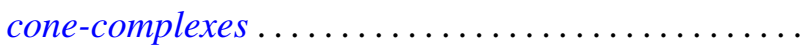

Richard R. Tucker, The $\delta^{2}$-process and related topics.... 